\title{
An Innovative Cellular Automata Technique for Mapping Cracking Pattern of Airport Pavement
}

\author{
Fucheng Yin ${ }^{1}$, Guangchun Zhou ${ }^{2}$, Edward Guo ${ }^{3}$, Shuang Zhang ${ }^{1, a}$ \\ ${ }^{1}$ Data Recovery Key Laboratory of Sichuan Province, Neijiang Normal University, Neijiang 641100, Sichuan, P.R. China; \\ ${ }^{2}$ School of Civil Engineering, Harbin Institute of Technology, China, 150090; \\ ${ }^{3}$ America, RSA, Atlantic City, 08405
}

\begin{abstract}
In this study, an innovative cellular automata (CA) technique was proposed for mapping cracking pattern of the airport pavement. The CA technique was developed to establish a numerical model describing the effect of boundary condition of pavement on zones (CA cells) within the pavement. A state function was used to describe the state values in the cells within the CA lattice. The correction coefficient principle is used as the criterion of zone similarity and the corresponding technique is proposed to find similar zones within and between pavements. Three pavement models, HRS, MRS and LRS, tested in FAA, USA, are set as the base pavements to map the cracking patterns of pavements with different sizes from the base pavements. The mapped cracking patterns of unseen pavements are empirically verified by referring to the relative experimental models.
\end{abstract}

\section{Introduction}

In the current research, three issues on structural engineering have been raised:

The first one is about the existing conventional analytical methods. In some cases, for example, for masonry and reinforced concrete as well as other complex and composite structures, an accurate prediction of structural response/behavior could not be achieved as long as global parameters of structural properties are used; besides, these structures have obvious variation in physics and configuration properties, which is generally treated as random factor. However, is the variation really totally random? In other words, is there any determinative content included in the random factor? If yes, how do we extract and model it?

The second one is about the existing experiment or test. As is well-known, no experiment is perfect, because the experimental error corresponding to the real situation is unavoidable. The problem is that these experimental data with error have not been fully used in the current analytical methods and design codes. Besides, these experimental data certainly include some useful information which is not discovered at present.

The third one is that artificial intelligence (AI) techniques are more and more applied to the civil engineering. These AI techniques such as neural networks, cellular automata, genetic algorithm and support vector can deal with highly non-linear and multiparameter engineering problems efficiently. The question is, to what extent can AI techniques be better than traditional analytical techniques to use experimental data

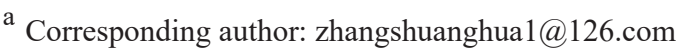

or to make analytical techniques closer to the real situation?

This study involves in the first and third issues related to airport pavements. The developed CA technique for mapping the cracking pattern of the pavement is based on two concepts(stiffness corrector and zone similarity) and two criteria (the criterion for matching similar zones within and between pavements and the criterion for mapping/compositing the cracking pattern of a pavement based on the tested cracking pattern of the pavement). Thus, it realizes a CA modeling method as well as the corresponding technique for directly mapping the cracking pattern of pavement from the tested results.

\section{The CA Technique for Mapping Cracking Pattern of Pavement}

The CA technique for mapping cracking pattern of pavement is based on the concept of zone similarity which actually came from a tested data mining [Zhou, 2003]. From the curves of ratios between the tested and FEA displacements at various measured points on the wall panel, it was found that these ratios approach to their individual stable values after the load reaches a certain level. Hence, these stable ratios were defined as the stiffness/strength correctors because they could be used to modify the global parameters of structural properties, such as the elastic modulus, so as to improve the FEA accuracy of the wall panels. Furthermore, from the contour plots of the correctors, it was found that the corrector distribution patterns in some zones are similar. It was conceptually verified that this zone similarity is 
related to similar boundary types and similar locations of zones. Thus, the concept of zone similarity and the criterion for matching similar zones within and between wall panels was proposed; the details can be found in References [1] and [2].

Cellular Automata is an artificial intelligent technique based on a discrete space-time lattice, introduced by von Neumann (1966). There are four parts in a CA model: the physical environment, the state of a cell, the neighborhoods of a cell and a local transition rule (Sarkar, 2000; Maerivoet and Moor, 2005). Fig. 1 shows two common CA models, the von Neumann model and the Moore model, and they have four and eight neighborhoods for a cell, respectively. These two models have been applied to model airport pavements as follows.

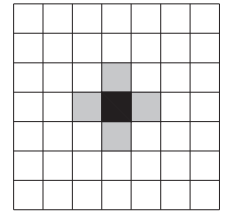

(a)

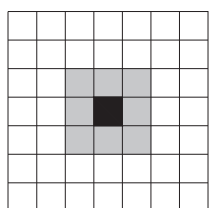

(b)
Fig. 1. The CA models: (a) the von Neumann model; (b) the Moore model

Fig. 1 is also used to represent the CA model of a pavement, in which a cell indicates a zone. For the state values of individual zones within a pavement, they can be calculated by Eq. (1) of the von Neumann model or Eq. (2) of the Moore model (Zhou, G. C. et al, 2002). The transition functions in Eq. (1) and Eq. (2) propagate the boundary effect into individual zones within the wallet.

$$
\begin{array}{cl}
L_{i, j}=L_{i, j-1}+\eta\left(1-L_{i, j-1}\right) & (i=1,2, \mathrm{~L}, M ; j=1,2, \mathrm{~L}, N) \\
R_{i, j}=R_{i, j+1}+\eta\left(1-R_{i, j+1}\right) & (i=1,2, \mathrm{~L}, M ; j=N, N-1, \mathrm{~L}, 1) \\
B_{i, j}=B_{i-1, j}+\eta\left(1-B_{i-1, j}\right) & (i=M, M-1, \mathrm{~L}, 1 ; j=1,2, \mathrm{~L}, N) \\
T_{i, j}=T_{i+1, j}+\eta\left(1-T_{i+1, j}\right) & (i=M, M-1, \mathrm{~L}, 1 ; j=N, N-1, \mathrm{~L}, 1) \\
L T_{i, j}=L T_{i-1, j-1}+\eta\left(1-L T_{i-1, j-1}\right) & (i=1,2, \mathrm{~L}, M ; j=1,2, \mathrm{~L}, N) \\
R T_{i, j}=R T_{i-1, j+1}+\eta\left(1-R T_{i-1, j+1}\right) & (i=1,2, \mathrm{~L}, M ; j=N, N-1, \mathrm{~L}, 1) \\
L B_{i, j}=L B_{i+1, j-1}+\eta\left(1-L B_{i+1, j-1}\right) & (i=M, M-1, \mathrm{~L}, 1 ; j=1,2, \mathrm{~L}, N) \\
R B_{i, j}=R B_{i+1, j+1}+\eta\left(1-R B_{i+1, j+1}\right) & (i=M, M-1, \mathrm{~L}, 1 ; j=N, N-1, \mathrm{~L}, 1)
\end{array}
$$

defined as follows: 0.0 for a free edge, 0.2 for a simply supported edge and 0.4 a fixed edge;

$\eta$ is the transition coefficient which is equal to 0.2 ;

$M$ and $N$ are the numbers of rows and columns of divided zones, respectively.

The state value $S_{i, j}$ of each cell is closely related to its four or eight adjacent cells and defined as the average effect from its four or eight neighborhoods, which is shown in Eq. (3) and Eq.(4).

$$
\begin{aligned}
& S_{i, j}=\frac{L_{i, j}+R_{i, j}+B_{i, j}+T_{i, j}}{4} \quad(i=1,2 \mathrm{~L}, M ; j=1,2, \mathrm{~L}, N) \\
& S_{i, j}=\frac{L_{i, j}+R_{i, j}+B_{i, j}+T_{i, j}+L T_{i, j}+R T_{i, j}+L B_{i, j}+R B_{i, j}}{8} \quad(i=1,2 \mathrm{~L}, M ; j=1,2, \mathrm{~L}, N)
\end{aligned}
$$

The past research results (Zhou, 2003) indicate that the cracking pattern/mode of wall panel is governed by the configuration of the structure, that is to say, the cracking mode of wall panel is closely related to the resulting effect of its boundary types and dimension. This just satisfies the CA function which can propagates the boundary constraint effect into individual zones within the wall panel. Therefore, a CA transition function and the corresponding formula are used to express the state of a zone. This state value reflects the effect of the boundaries and zone locations on the zone. In other words, this state value quantifies the effect of the structural configuration on the zone. Hence, a cellular automata technique for automatically and mechanically matching similar zones was developed. This CA criterion for matching similar zones needs to calculate 8 difference values corresponding to 8 different orientations of two zones with their neighborhoods, as shown in Fig. 2. Then the minimum one in the eight difference values is determined as a so-called ratio between two zones. Similarly, $N$ so-called ratios are obtained for a zone on a panel to $N$ zones divided on the other panel. Again, the minimum one in these $N$ so-called ratios is determined. Finally, two zones corresponding to the minimum value among $\mathrm{N}$ values are defined as similar zones.

Correction coefficient, which ranges from -1 to 1 , is an index about the corrective degree between two variables. The closer the correction coefficient approaches to 1 , the better the linear relationship between the two variables is. Accordingly we can estimate the 
zone similarity between the new plate and the base plate by calculating the correction coefficients of corresponding zones in the two plates. In order to estimate the zone similarity between the new plate and the base plate, we use the Moore neighborhood model, which is expressed as follows:

$$
\begin{gathered}
\bar{S}_{i, j}^{\text {new }}=\frac{1}{9} \sum_{u=-1}^{1} \sum_{v=-1}^{1} S_{i+u, j+v}^{\text {new }} \\
\bar{S}_{m, n}^{\text {base }}=\frac{1}{9} \sum_{u=-1}^{1} \sum_{v=-1}^{1} S_{m+u, n+v}^{\text {base }}
\end{gathered}
$$

Where $E_{i, j \rightarrow \text { new }}^{m, n \rightarrow \text { base }}$ is the maximum correction coefficients of corresponding zones in the new plate and the base plate; $i, j$ are zone position in the new plate, respectively; $m, n$ are zone position in the base plate, respectively; $S_{m, n}^{\text {base }}$ is state values of the base plate; $\bar{S}_{i, j}^{\text {new }}$ is average state value of any zone and all its neighborhood zones in the new plate; $\bar{S}_{m, n}^{\text {base }}$ is the average state value of any zone and all its neighborhood zones in the base plate.

We can get the maximum correction coefficients of corresponding zones in the new plate and the base plate from Eq. (3-3) - Eq. (3-5); then the zone $(m, n)$ in the base plate can be defined as the similar zone of the zone $(i, j)$ in the new plate.

Once similar zones are matched, the rule for identifying the cracking zones within the pavement can be proposed as follows: if two zones are similar zones under the same loading, these two zones have the same behavior, in other words, if a zone is cracked, then, its similar zone is also cracked.

By summarizing all the results above, a CA technique for mapping the cracking pattern of the pavement can be formed as shown in Fig. 4: the first step is to use the proposed CA modeling method, mesh both unseen and the tested pavements to get their CA lattices, and then the cracking pattern of the tested pavement is projected to its CA lattice; the second step is to use the CA rule for matching similar zones and match similar zones between the two pavements; the third step is to use the rule for identifying cracking zones to map the cracking pattern of the unseen pavement.

\begin{tabular}{l} 
The CA modeling of the tested \\
pavement as the base pavement and \\
proiect its cracking pattern to the CA \\
\begin{tabular}{l} 
The CA modeling of the unseen \\
\hline $\begin{array}{l}\text { Matching zone similarity between the } \\
\text { base and unseen pavements }\end{array}$ \\
Mapping the cracking pattern of the \\
unseen pavement based on the \\
cracking pattern of the base
\end{tabular} \\
\hline
\end{tabular}

\section{Case Study on Mapping Cracking Pattern of Pavement}

In the following some examples of concrete pavements are presented to validate the $\mathrm{CA}$ technique proposed in this study. Three examples are the pavements tested in NAPTF, FAA ().

Example: The pavement HRS is taken as the base pavement to map the cracking patterns of a smaller and a larger unseen pavements. Similarly, the smaller and larger pavements are all the same with the base pavement except for their lengths and widths. The mapped results are shown in Fig. 7.

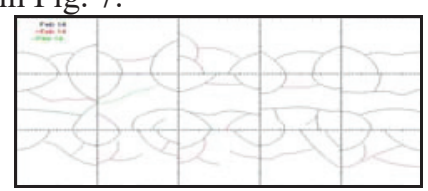

Fig. 5 The cracking pattern of the tested pavement

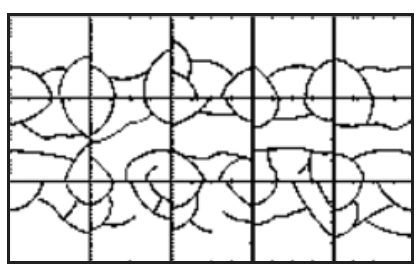

Fig. 6 The cracking pattern on CA lattice

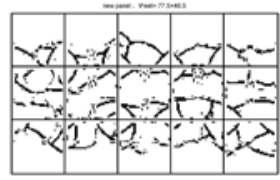

(a)

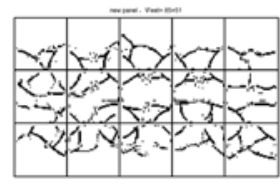

(b)

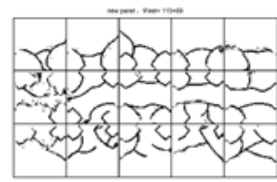

(b)

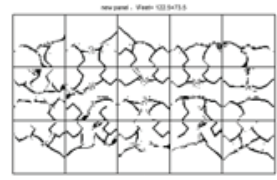

(d)
Fig. 7 The mapped cracking patterns of two unseen pavements from Pavement HRS

(a) The CA technique needs further improvement because we do not know how accurately it reflects real 
performance of pavements.(b)The thickness needs further modeling in the CA numerical mode of pavement.(c)The configuration of pavement needs further modeling in the CA numerical mode of pavement.

The six examples show that two new concepts, two rules, one CA modeling method and one $\mathrm{CA}$ technique for mapping the cracking pattern of the structure are valid.

Because all these contents are quite new, they certainly have some problems to be further studied, as shown on the screen. But the results achieved in this study are significant, I think.

First the CA technique we have developed can save research funds; in my study on masonry wall panels, at least $40 \%$ fund could have been saved if the CA technique had been used.

Second, the CA technique can obtain more knowledge and comprehension on the problems from the test data.

Third, the CA technique can be directly used by design engineers besides researchers.

\section{Conclusions}

The proposed CA technique for mapping cracking pattern of pavement 1.Provides a new way in which the failure/cracking pattern of a pavement is directly "predicted", only based on the failure/cracking pattern of the base pavement.2.Could considerably save research fund for test, because a few typical tested pavements could be used to produce the "test results" of unseen pavements.3.Explores the new application of the existing experimental data of full-scale structural models.4.The pattern matching techniques using cellular automata, developed in this study, can replace the conventional finite element analysis and are capable of predicting the failure patterns of laterally-loaded masonry wall panels with various boundary conditions.5.The boundary condition and the variation in masonry properties at various locations within the panel, as discussed in the authors' previous publications, play a key role in determining the failure patterns of laterally-loaded masonry panels. 6. The CA technique introduced in this paper can be used as reference for analysis and design of masonry panels more confidently.7.Techniques developed in this paper can be used as an artificial experimental environment replacing some of the physical tests for masonry panels, which could be very expensive.

\section{Acknowledgments}

This work presented in this paper is supported by The Sichuan Province Department of Science and Technology under Grant 14ZA0243; The authors would like to thank Harbin Institute of Technology, China, the FAA and the SRA, USA, for their supports to this project. This research has been supported financially by the Yoh Foundation, USA.

\section{References}

1. Anthony, T.C., Goh et al. (1995). "Estimation of lateral wall movements in braced excavations using neural networks." Can. Geotech. J., 32, 1059-1064

2. Arslan, A., and Ince, R. (1996). "Neural networkbased design of edge-supported reinforced concrete slabs." Structural Engineering Review, 8(4), 329-335

3. Ashraf, M., Elazouni et al. (1997). "Estimating resource requirements at conceptual stage using neural networks." Journal of Computing in Civil Engineering, 11(4)

4. Chen, S., and Shan, K. (1992). "Neural networks in dynamic analysis of bridges.' Proc., 8th Annual Conference of Computing in Civil Engineering, ASCE, 1058-1065

5. Drysdale, R.G., and Hamid, A.A. (1984). "Tension failure criteria for plain concrete masonry." Journal of Structural Engineering, ASCE, 110(2), 228-244

6. Flood, I. and Kartam N. (1994). "Neural networks in civil engineering. I: Principles and understanding. II: Systems and application". Journal of Computing in Civil Engineering, 8(2)

7. Garrett, J.H., Jr., Ghaboussi, J., Wu, X., and Ranjithan, S. (1992). "Neural networks and their application to civil engineering." Expert systems in civil engineering-Knowledge representation, R. H. Allen, ed., Expert Systems Committee of ASCE, New York

8. Ghaboussi, J., Garrett, J.H., Jr., and Wu, X. (1991). "Knowledge-based modeling of material behavior with neural networks.' J. Eng. Mech., 117(1), 132153

9. Haykin, S. (1994). "Neural networks: a comprehensive foundation." New York, Macmillan

10. Hsu, D.S., Yeh, I.C., and Lian, W.T. (1993). "Artificial neural damage detection of existing structure.' Proc., 3rd ROC and Japan Seminar on Natural Hazards Mitigation, Tainan, 423-436

11. Larry, M. et al. (1997). "Finite-element mesh generation using self-organizing neural networks." Microcomputers in Civil Engineering, 12, 233-250

12. Liu, L.P., Tang, D.X., and Zhai, X.M. (2006). "Failure criteria for grouted concrete block masonry under biaxial compression." Advances in Structural Engineering, 9(2), 229-239

13. Mathew, A., Kumar, B., Sinha, B.P., and Pedreschi, R.F. (1999). "Analysis of masonry panel under biaxial bending using ANNs and CBR." Journal of Computing in Civil Engineering, 170-177

14. Mukherjee, A. et al. (1996). "Prediction of buckling load of columns using artificial neural networks." Journal of Structural Engineering, 122(11)

15. Rafiq, M.Y., Bugmann, G., and Easterbrook, D.J. (1998). "Artificial neural networks for modelling some of the activities of the conceptual stage of the design process." ASCE Computing Congress, 1998

16. Sarkar, P. (2000). "A brief history of cellular automata." ACM Comput. Surveys, 32(1), 80-107

17. Smith, M. (1994). "Neural networks for statistical modeling." Van Nostrand Reinhold, NY, 235

18. Maerivoet, S., and Moor, B.D. (2005). "Cellular automata models of road traffic." Physics Reports, $419,1-64$ 
19. Tsai, C.H., and Hsu, D.S. (1997). "Reinforced concrete structural damage diagnosis by using artificial neural network." Proc., Intelligent Information System, IEEE Computer Society, Los Alamitos, Calif., 149-155

20. Tsai, C.H., and Hsu, D.S. (2002). "Diagnosis of reinforced concrete structural damage base on displacement time history using the backpropagation neural network technique." Journal of Computing in Civil Engineering, 16(1), 49-58

21. Von Neumann, J. (1966). Theory of Self Reproducing Automata, Burks, A., ed., University of Illinois Press, Champaign, Ill. 305-317

22. Zhou, G.C. (2002). "Application of stiffness/strength corrector and cellular automata in predicting response of laterally loaded masonry panels." Ph.D. thesis, University of Plymouth, U.K

23. Zhou, G.C., Rafiq, M.Y., Bugmann, G.C., and Easterbrook, D.J. (2006). "Cellular automata model for predicting the cracking pattern of laterally loaded masonry wall panels." Journal of Computing in Civil Engineering, 20(6), 400-409

24. Zhou, G.C., Rafiq, M.Y., Easterbrook, D.J. and Bugmann, G.C. (2003). "Application of cellular automata in modeling laterally loaded masonry panel boundary effects." Masonry Int., 16(3), 104-114 